PROCEEDINGS OF THE

AMERICAN MATHEMATICAL SOCIETY

Volume 134, Number 2, Pages 343-349

S 0002-9939(05)08043-3

Article electronically published on August 12, 2005

\title{
A FINITELY PRESENTED GROUP WITH UNBOUNDED DEAD-END DEPTH
}

\author{
SEAN CLEARY AND TIM R. RILEY
}

(Communicated by Alexander N. Dranishnikov)

\begin{abstract}
The dead-end depth of an element $g$ of a group $G$, with respect to a generating set $\mathcal{A}$, is the distance from $g$ to the complement of the radius $d_{\mathcal{A}}(1, g)$ closed ball, in the word metric $d_{\mathcal{A}}$ defined with respect to $\mathcal{A}$. We exhibit a finitely presented group $G$ with a finite generating set with respect to which there is no upper bound on the dead-end depth of elements.
\end{abstract}

\section{INTRODUCTION}

We explore the behavior of geodesic rays and the geometry of balls in Cayley graphs of finitely generated groups. An element $g$ in a group $G$ is defined by Bogopol'skil [3] to be a dead end with respect to a generating set $\mathcal{A}$ (which will always be finite in this article) if it is not adjacent to an element further from the identity; that is, if a geodesic ray in the Cayley graph of $(G, \mathcal{A})$ from the identity to $g$ cannot be extended beyond $g$. Dead-end elements occur in a variety of settings. Bogopol'skiu showed that $\mathrm{SL}_{2}(\mathbb{Z})$, presented by $\left\langle x, y \mid x^{4}, y^{6}, x^{-2} y^{3}\right\rangle$, has dead ends with respect to $\{x, y\}$ and that $\left\langle x, y \mid x^{3}, y^{3},(x y)^{k}\right\rangle$, with $k \geq 3$, has dead ends with respect to $\{x, y\}$ but not $\{x, y, x y\}$. Fordham 8 found dead ends in Thompson's group $F$, presented by $\left\langle x_{0}, x_{1} \mid\left[x_{0} x_{1}{ }^{-1}, x_{0}{ }^{-1} x_{1} x_{0}\right],\left[x_{0} x_{1}{ }^{-1}, x_{0}{ }^{-2} x_{1} x_{0}{ }^{2}\right]\right\rangle$, with respect to the standard finite generating set $\left\{x_{0}, x_{1}\right\}$. In Lemma 4.19 of 4 Champetier shows that presentations $\langle\mathcal{A} \mid \mathcal{R}\rangle$ satisfying the $C^{\prime}(1 / 6)$ small cancellation condition have no dead ends with respect to $\mathcal{A}$. In general, generating sets can be contrived with respect to which there are dead ends; for example, $\mathbb{Z}$ has dead ends with respect to the generating set $\left\{a^{2}, a^{3}\right\}$. Many of these examples are discussed in IV.A.13,14 of de la Harpe [7, where there is also an exercise due to Valette concerning further examples.

Dead ends differ in their severity in the following sense. We define the depth, with respect to $\mathcal{A}$, of $g \in G$ as the distance in the word metric $d_{\mathcal{A}}$ between $g$ and the complement in $G$ of the closed ball $B_{g}$ of radius $d_{\mathcal{A}}(1, g)$ centered at 1 . If $G \backslash B_{g}$ is empty we define the depth of $g$ to be $\infty$. So $g \in G$ is a dead end when its depth is at least 1 . Cleary and Taback [5] showed that, with respect to $\left\{x_{0}, x_{1}\right\}$, the dead-end elements in Thompson's group $F$ are all of depth 2.

Received by the editors July 26, 2004 and, in revised form, September 18, 2004.

2000 Mathematics Subject Classification. Primary 20F65.

Key words and phrases. Dead-end depth, lamplighter.

Support for the first author from PSC-CUNY grant \#65752 is gratefully acknowledged.

Support for the second author from NSF grant 0404767 is gratefully acknowledged. 
Cleary and Taback [6] exhibited wreath products, such as the lamplighter group $\mathbb{Z}_{2} \imath \mathbb{Z}$, with unbounded dead-end length with respect to certain standard generating sets. Independently, Erschler observed that $\mathbb{Z}_{2} \prec \mathbb{Z}$ provides an example resolving a closely related question of Bowditch (Question 8.4 in Bestvina's problem list 2]): let $\Gamma$ be the Cayley graph of an infinite finitely generated group; does there exist $K>0$ such that for all $R>0$ and all vertices $v \in \Gamma-B(R)$ there is an infinite ray from $v$ to $\infty$ which does not enter $B(R-K)$ ?

However, these wreath product examples are not finitely presentable. So a natural question, asked by Bogopol'skiı [3], is whether, given a finitely presentable group and a finite generating set $\mathcal{A}$, there is an upper bound on dead-end depth with respect to $\mathcal{A}$. Bogopol'skii [3] showed that there is always such a bound in the case of hyperbolic groups. We answer this question in the negative with the main result of this paper:

Theorem 1.1. There is a finitely presentable group $G$ that has a finite generating set $\mathcal{A}$ with respect to which $G$ has unbounded dead-end depth. We take $G$ to be the group presented by

$$
\mathcal{P}:=\left\langle a, s, t \mid a^{2}=1,\left[a, a^{t}\right]=1,[s, t]=1, a^{s}=a a^{t}\right\rangle
$$

and consider the generating set

$$
\mathcal{A}:=\{a, s, t, a t, t a, \text { ata }, a s, s a, a s a\} .
$$

We denote the commutator $a^{-1} b^{-1} a b$ by $[a, b]$ and the conjugate $b^{-1} a b$ by $a^{b}$.

The lamplighter group $\mathbb{Z}_{2} \imath \mathbb{Z}$, which has presentation

$$
\left\langle a, t \mid a^{2},\left[a, a^{t^{i}}\right], \forall i \in \mathbb{Z}\right\rangle,
$$

is a subgroup of $G$. Removing the defining relation $a^{2}$ from $\mathcal{P}$ gives Baumslag's remarkable example [1] of a finitely presented metabelian group containing $\mathbb{Z} \imath \mathbb{Z}$ and thus a free abelian subgroup of infinite rank.

The reference to a specific finite generating set in the theorem is important as the following issues are unresolved 11 Is the property of having unbounded deadend depth an invariant of finitely generated groups? That is, does it depend on the choice of finite generating set? Moreover, is this property a quasi-isometry invariant? Indeed, does the group $G$ defined above have unbounded dead-end depth with respect to all finite generating sets?

\section{The LAMPLIGHTER GRID MODEL FOR $G$}

The group $\mathbb{Z}_{2} \nmid \mathbb{Z}$ was named the lamplighter group by Cannon (see Parry [9]) on account of the following faithful, transitive action on $\mathcal{P}_{\text {fin }}(\mathbb{Z}) \times \mathbb{Z}$. Here, $\mathcal{P}_{\text {fin }}(\mathbb{Z})$ denotes the set of finite subsets of $\mathbb{Z}$; that is, finite configurations illuminated among a string of lamps indexed by $\mathbb{Z}$. The second factor denotes the location of a lamplighter (or a cursor) in the string of lamps. The action of the generator $t$ (of the presentation $\mathcal{P}$ in the introduction) is to increment the location of the lamplighter by one, and the action of $a$ is to toggle the lamp at the current location of the lamplighter between on and off.

\footnotetext{
${ }^{1}$ Remark added August 2004. It has since been shown [10] that $\langle a, t, u| a^{2}=1,[t, u]=$ $\left.1, a^{t}=a^{u} ; \forall i \in \mathbb{Z},\left[a, a^{t^{i}}\right]\right\rangle$ has unbounded depth dead ends with respect to one finite generating set, but no dead ends at all with respect to another.
} 
We will develop a lamplighter model for $G$ and describe a faithful, transitive left action of $G$ on $\mathcal{P}_{\text {fin }}(\mathbb{Z}) \times \mathbb{Z}^{2}$, to gain insights into its metric properties. Killing $a$ retracts $G$ onto the subgroup $\langle s, t\rangle \cong \mathbb{Z}^{2}$, and gives the location of a lamplighter among the lattice points of the infinite grid illustrated in Figure 1 For reasons that will become apparent later, we draw the grid skew and we add dashed lines to subdivide it into triangles. As before, elements of $\mathcal{P}_{\text {fin }}(\mathbb{Z})$ determine which of a $\mathbb{Z}$-indexed string of lamps are illuminated. We arrange these lamps on the $t$-axis of the grid, and at every lattice point there is a button. The actions of $s$ and $t$ are to move the lamplighter one unit in the $s$ and $t$ directions, respectively. The action of $a$ is to "press the button" at the location of the lamplighter, setting off signals that propagate toward the lamps by taking all possible shortest paths to the $t$-axis in the 1-skeleton of the triangular lattice (each side of each triangle is given length 1) of Figure 1. At each vertex en route to the $t$-axis, each signal will bifurcate into two signals. Each lamp then switches between on and off once for every signal it receives. A lamp may receive many signals, and its end state depends on its initial state and whether the total number of signals received is even or odd. We note that the signals can travel along the solid grid lines and also along the dashed lines of the triangular grid, but that the lamplighter can move only along the solid lines of the rhombic grid.

The manner in which these signals split as they propagate towards the $t$-axis leads to a connection with Pascal's triangle modulo 2. We can understand the action of $a$ as follows. Suppose the current location of the lamplighter is given with $s$ - and $t$-coordinates of $(p, q)$. Define $\varepsilon=1$ if $p \geq 0$ and $\varepsilon=-1$ otherwise. Then for $r \in\{0,1, \ldots, p\}$, the lamp at position $t^{q+\varepsilon r}$ is toggled between on and off when there is a 1 in the $r$-th entry of row $p$ of Pascal's triangle mod 2.

As an example, Figure 1 gives the action of $a$ when the lamplighter, denoted by an asterisk, is located at $(6,-4)$ and the lights in positions $-4,-2,-1,2,3,4$ are illuminated, as shown in the left diagram. Pascal's triangle mod 2 is overlaid in the middle diagram, and the right diagram shows the result of the signals on the lamp configuration.

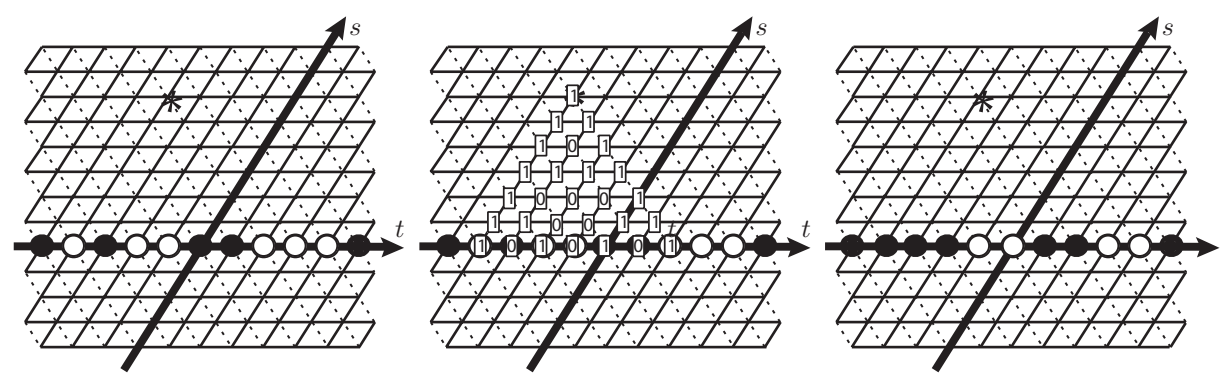

Figure 1. An example of the action of $a$. The left diagram shows $g(\emptyset,(0,0))$ and the right diagram shows $a g(\emptyset,(0,0))$, where $g=$ $s^{6} a t^{-2} a t^{-1} a t^{-3} a t^{-1} a t^{-1} a t^{4}$. Along the $t$ axis, open circles indicate illuminated lamps and filled-in circles indicate lamps which are off.

To verify that we have a well-defined action of $G$ it suffices to check that $a^{2},\left[a, a^{t}\right]$, and $[s, t]$ all act trivially and that the actions of $a a^{t}$ and $a^{s}$ agree. This and the proof that the action is transitive are straightforward, and we leave them to the reader. To 
show that the action is faithful we suppose $g \in G$ satisfies $g(\emptyset,(0,0))=(\emptyset,(0,0))$ and we will check that $g=1$ in $G$. Let $w$ be a word representing $g$. Consider the rhombic grid with the lights all off and the lamplighter at $(0,0)$. Reading $w$ from right to left determines a path for the lamplighter starting and finishing at $(0,0)$, in the course of which buttons are pressed: each letter $s^{ \pm 1}, t^{ \pm 1}$ moves the lamplighter, and each $a^{ \pm 1}$ toggles the lamp at the location of the lamplighter. Because of the

relations $[s, t]=1$ and $a a^{t}=a^{s}$ we may alter $w$ to another word $w^{\prime}$ such that $w=w^{\prime}$ in $G$ and such that in the course of the path determined by $w^{\prime}$, the only buttons that are pressed are on the $t$-axis. Then, by removing inverse pairs from $w^{\prime}$ and applying the relation $[s, t]=1$ we can find a word $w^{\prime \prime}$ with $w^{\prime}=w^{\prime \prime}$ in $G$ such that the path determined by $w^{\prime \prime}$ does not leave the $t$-axis. But then $w^{\prime \prime}$ represents an element of the subgroup $\langle a, t\rangle=\mathbb{Z}_{2} \imath \mathbb{Z}$ in $G$ and as $w^{\prime \prime}$ illuminates no lights and returns to $(0,0)$, we deduce that $g=1$.

\section{Proof of the theorem}

We define maps $\mathcal{I}: G \rightarrow \mathcal{P}_{\text {fin }}(\mathbb{Z})$ and $\mathcal{L}: G \rightarrow \mathbb{Z}^{2}$ by

$$
(\mathcal{I}(g), \mathcal{L}(g))=g(\emptyset,(0,0)) .
$$

So if initially the lamplighter is at the origin and no lamps are lit, then $\mathcal{I}$ gives the lamps illuminated and $\mathcal{L}$ gives the location of the lamplighter after the action of $g$. We define $H_{n}$ to be the subset of $\mathbb{Z}^{2}$ of lattice points in (and on the boundary of) the hexagonal region of the grid with corners at $( \pm n, 0),(0, \pm n),(n,-n),(-n, n)$. This is illustrated in Figure 2 in the case $n=4$, shaded medium and dark gray.

In the following proposition we determine the distance of various group elements from the identity in the word metric with respect to

$$
\mathcal{A}=\{a, s, t, a t, t a, a t a, a s, s a, a s a\} .
$$

The crucial feature of this generating set is that the button at a vertex the lamplighter is leaving or arriving at can be pressed with no additional cost to word length. Thus for $g \in G \backslash\{a\}$, the distance $d_{\mathcal{A}}(g, 1)$ is the length of the shortest path in the rhombic grid that starts at $(0,0)$, finishes at $\mathcal{L}(g)$, and such that pressing some of the buttons at the vertices visited produces the configuration $\mathcal{I}(g)$ of illuminated bulbs.

Proposition 3.1. Fix $n \in\{1,2, \ldots\}$ and suppose $g \in G$. If $\mathcal{I}(g) \subseteq\{-n, \ldots, n\}$ and $\mathcal{L}(g) \in H_{n}$, then $d_{\mathcal{A}}(1, g) \leq 4 n$.

Proof. Define $(p, q)=\mathcal{L}(g)$. So $p$ and $q$ are the $s$ - and $t$-coordinates, respectively, of the position of the lamplighter.

Let $T_{n} \subset \mathbb{Z}^{2}$ be the set of lattice points in, and on the boundary of, the triangular region with corners $(0,0),(0,-n),(n,-n)$. Of the two triangular regions in Figure 2 shaded dark gray, $T_{n}$ is the one above the $t$-axis (shown in the case $n=4$ ).

First, we assume $(p, q) \in T_{n}$. Let the lamplighter travel along the $t$-axis to $(0, n)$, then along the $t$-axis to $(0,-n)$, then along the $t$-axis to $(0, q)$, and then parallel to the $s$-axis to $(p, q)$. The total number of edges traversed is at most $4 n$. When following this path the lamplighter visits all the lamps that are to be illuminated, and in the course of visiting can press the required buttons at no additional cost because of the choice of generating set. So $d_{\mathcal{A}}(1, g) \leq 4 n$.

Next, we assume $\mathcal{L}(g) \in H_{n} \backslash T_{n}$ and $p \geq 0$. Then $\mathcal{L}(g)$ is in, or on the boundary of, the rhombic region above the axis (shaded medium gray in Figure 2). We can 


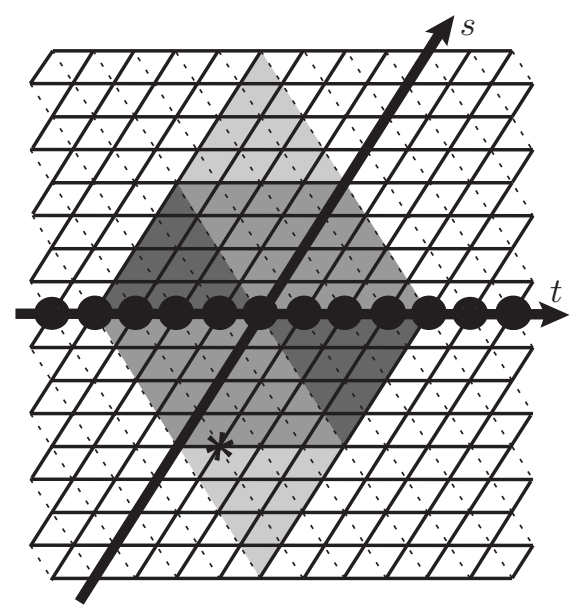

Figure 2. Diamond, hexagonal, and triangular regions in the lamplighting grid.

have the lamplighter follow a path $\phi$, first along the $t$-axis to $(0,-n)$, then back along the $t$-axis to $(0,-p)$, then parallel to the $s$-axis to $(p,-p)$, then parallel to the $t$-axis to $(p, n-p)$, and then parallel to the $t$-axis back to to $(p, q)$. These five arcs have length $n, n-p, p, n$ and $n-p-q$, respectively, totalling $4 n-p-q$, which is less than $4 n$ because $p>-q$.

We now show that pressing some combination of buttons on $\phi$ achieves the configuration $\mathcal{I}(g)$ of illuminated lamps. The lamps in positions $-n,-n+1, \ldots$, $-p-1$ can be illuminated as required when traversing the second arc of $\phi$. The lamps in positions $-p,-p+1, \ldots, n$ determine a sequence of $n+p+1$ zeros and ones which make up the base of a trapezoid of zeros and ones as analyzed in Lemma 3.2 . Overlaying this trapezoid on the grid, we find that its left and top sides follow the third and fourth arcs of $\phi$. Pressing the buttons at the locations of the summits (defined in Lemma 3.2) in the trapezoid illuminates the required lamps. This is because the trapezoid is constructed in such a way that each entry equals the total number of signals (see Section 2) mod 2 reaching its location when the buttons at the summits are all pressed. In other words, the trapezoid is the overlay $(\bmod 2)$ of a number of copies of Pascal's triangle, one for each summit; so, for example, the trapezoid in Figure 3 is the overlay of five triangles, with heights 1,2, 4, 5 and 5.

Similar arguments (with the paths rotated through $180^{\circ}$ about the origin) show that $d_{\mathcal{A}}(1, g) \leq 4 n$ when $p<0$.

To understand the possible lightbulb configurations obtainable from these paths which go first in the $s$-direction and then in the $t$-direction, we consider trapezoidal regions modelled on Pascal's triangle. As in Pascal's triangle, we understand entries outside the trapezoidal region to be zero.

Lemma 3.2. Suppose $S$ is a sequence of $m$ zeros and ones, and suppose $r \in$ $\{1,2, \ldots, m\}$. There is a trapezoid consisting of zeros and ones (entries) satisfying the following. The entries are arranged in $r$ rows with the bottom row $S$ and all the other rows containing one fewer entry than that below it (as in Figure 31). Every entry is the sum of the two entries immediately above it mod 2, with the possible 
exception of some entries in the top row and at the left ends of the rows. We call these exceptional entries summits.

Proof. We use induction on $r$. When $r=1$ there is nothing to prove. For the induction step, we assume the lowest $r$ rows have been constructed as per the hypotheses. We add the next row one entry at a time starting from the right-hand side. We select each entry so as to ensure that of the two entries immediately below it, the one to the right has the property that it is the sum of the two entries immediately above it mod 2. This ensures that only entries in the left side the trapezoid and in the $(r+1)$-st row can fail to equal the sum of the two entries immediately above them, mod 2 .

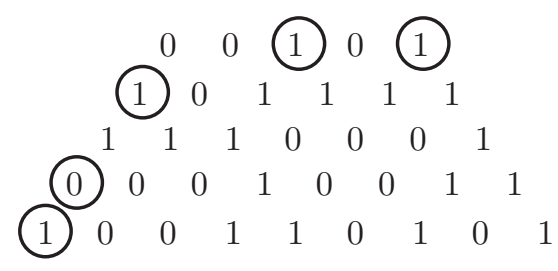

Figure 3. A trapezoid of ones and zeros in which every entry other than those circled (called summits) is the sum of the two entries above it $\bmod 2$.

Proposition 3.3. Fix $n \in\{1,2, \ldots\}$. Suppose $g \in G$ satisfies $\{-n, n\} \subseteq \mathcal{I}(g)$ and $\mathcal{L}(g)=(0,0)$. Then $d_{\mathcal{A}}(1, g) \geq 4 n$.

Proof. Consider the diamond-shaped region $D_{n}$ with vertices at $(0, \pm n),(2 n,-n)$, $(-2 n, n)$. In Figure 2, this region in the case $n=4$ is the union of all the gray shaded regions. In order to illuminate the lamp at $(0, n)$, the lamplighter must press a button at a vertex either on or to the right of one of the following two lines: that through $(-2 n, n)$ and $(0, n)$, and that through $(0, n)$ and $(2 n,-n)$. So the lamplighter must visit a vertex $v$ on one of these two lines. Likewise, for the lamp at $(0,-n)$ to be lit, a vertex $v^{\prime}$ on the line through $(-2 n, n)$ and $(0,-n)$ or on the line through $(0,-n)$ and $(2 n,-n)$ must be visited.

A path that starts and finishes at $(0,0)$ and visits two such vertices must traverse at least $4 n$ edges in the rhombic grid. This can be verified by calculation according to locations of $v$ and $v^{\prime}$. We will assume that neither $v$ nor $v^{\prime}$ is below the $t$-axis. The cases this excludes are analogous or easier and we leave them to the reader. Now $v=(k, n-k)$ and $v^{\prime}=\left(k^{\prime},-n\right)$ for some $0 \leq k, k^{\prime} \leq 2 n$. The length of the path is at least the sum of distances from $(0,0)$ to $v$, from $v$ to $v^{\prime}$, and from $v^{\prime}$ to $(0,0)$ in the rhombic grid. The distance is at least $L=(k+|n-k|)+\left(\left|k^{\prime}-k\right|+2 n-k\right)+\left(k^{\prime}+n\right)$. When $0 \leq k \leq n$ we find $L=4 n+2\left(k^{\prime}-k\right)$ if $k^{\prime} \geq k$ and $L=4 n$ if $k^{\prime} \leq k$. When $n<k \leq 2 n$, we find $L=2 n+2 k^{\prime}$ if $k^{\prime} \geq k$ and $L=2 n+2 k$ if $k^{\prime}<k$. So in all these cases $L \geq 4 n$, as required.

Proof of Theorem 1.1. Define $g_{n}:=t^{n} a t^{-2 n} a t^{n}=t^{n-1}(t a) t^{-2 n}(a t) t^{n-1}$. Then $d_{\mathcal{A}}\left(1, g_{n}\right) \leq 4 n$ and so by Proposition 3.3 we see $d_{\mathcal{A}}\left(1, g_{n}\right)=4 n$ because $g_{n}(\emptyset,(0,0))$ $=(\{-n, n\},(0,0))$. It follows from Proposition 3.1 that if $w$ is a word representing an element $g$ with $d_{\mathcal{A}}(1, g)>4 n$, then the lamplighter's path determined by $w$ (reading right to left and with the lamplighter initially at the origin) must leave 
$H_{n}$. But $\mathcal{L}\left(g_{n}\right)=(0,0)$, and $H_{n}$ contains the closed ball of radius $n$ about $(0,0)$ in the rhombic grid. So if $v_{n}$ is a word representing $g_{n}$ and $u_{n} v_{n}$ a word representing an element $h_{n}$ with $d_{\mathcal{A}}\left(1, h_{n}\right)>4 n$, then the length of $u_{n}$ is more than $n$. So, with respect to $d_{\mathcal{A}}$, the distance from $g_{n}$ to the complement of the radius $d_{\mathcal{A}}\left(1, g_{n}\right)$ ball is more than $n$. Thus, with respect to $\mathcal{A}$, the dead-end depth of $g_{n}$ is at least $n$ and $G$ has unbounded dead-end depth.

\section{ACKNOWLEDGMENT}

We began discussing the ideas in this paper at the 2004 Cornell Topology Festival. We are grateful to the organizers for their hospitality. We also thank Oleg Bogopol'skir for an account of the history of dead-end depth and the anonymous referee for a careful reading.

\section{REFERENCES}

[1] Gilbert Baumslag. A finitely presented metabelian group with a free abelian derived group of infinite rank. Proc. Amer. Math. Soc., 35:61-62, 1972. MR0299662 (45:8710)

[2] Mladen Bestvina. Questions in geometric group theory. www.math.utah.edu/ bestvina/ eprints/questions.dvi.

[3] O. V. Bogopol'skiř. Infinite commensurable hyperbolic groups are bi-Lipschitz equivalent. Algebra and Logic, 36(3):155-163, 1997. MR1485595 (98h:57002)

[4] Christophe Champetier. Propriétés statistiques des groupes de présentation finie. Adv. Math., 116(2):197-262, 1995. MR.1363765 (96m:20056)

[5] Sean Cleary and Jennifer Taback. Combinatorial properties of Thompson's group F. Trans. Amer. Math. Soc., 356(7):2825-2849 (electronic), 2004. MR2052598 (2005b:20074)

[6] Sean Cleary and Jennifer Taback. Dead end words in lamplighter groups and other wreath products. Quarterly Journal of Mathematics, to appear.

[7] Pierre de la Harpe. Topics in geometric group theory. Chicago Lectures in Mathematics. University of Chicago Press, Chicago, IL, 2000. MR.1786869 (2001i:20081)

[8] S. Blake Fordham. Minimal Length Elements of Thompson's group F. Ph.D. thesis, Brigham Young Univ., 1995.

[9] Walter Parry. Growth series of some wreath products. Trans. Amer. Math. Soc., 331(2):751759, 1992. MR1062874(92h:20061)

[10] Tim R. Riley. The unbounded depth dead end property is not a group invariant, 2004. www.math.yale.edu/users/riley.

Department of Mathematics, The City College of New York, City University of New York, New York, New York 10031

E-mail address: cleary@sci.ccny.cuny.edu

Department of Mathematics, Yale University, 10 Hillhouse Avenue, P.O. Box 208283, New Haven, Connecticut 06520-8283

E-mail address: tim.riley@yale.edu

Current address: Department of Mathematics, Cornell University, Ithaca, New York 148534201 